\title{
Airport Information Sharing Concept Architecture
}

\section{Rafael Apaza, NASA Glenn Research Center, Cleveland, Ohio}

Antonio Correas, Skymantics, Washington, DC

April 18-20, 2017 


\section{Outline}

1. Introduction

2. Project Description

3. Airport Architecture Concept

4. Requirements Development

5. Use Cases and Information Flows

6. Future Work and Conclusion 


\section{Introduction}

- Statistics collected by US DOT (2016):

- 23,911comercial flights daily

- 2,246,004 passengers fly every day

- 8,727,691 commercial flights in 2015

$-2.1 \%$ annual growth over next 20 yrs. (FAA)

- Today's airports are complex operations that depend on the efficient and timely execution of many different functions by stakeholders.

- There is an increasing need for timely exchange of information among all service providers to enable higher efficiency, safety and economic gains.

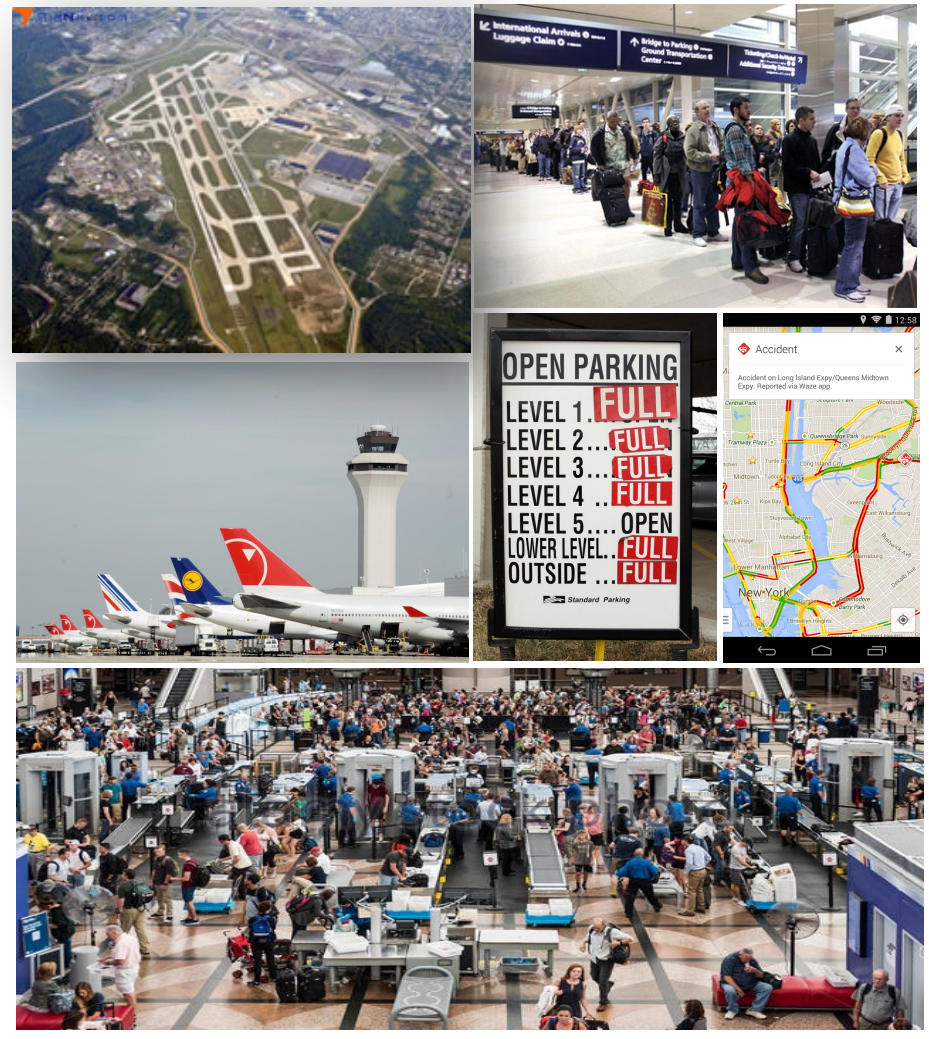




\section{Project Description}




\section{Airport Information Sharing Architecture}

Develop an airport information sharing architecture that enables seamless exchange of timely information among stakeholders for the improvement of NAS operations

\section{Challenge: Increase airport real time information exchange among stakeholders}

\section{Objectives}

- Increase airport capacity

- Improve gate turnaround time

- Improve on-time departures

- Improve NAS efficiency, capacity and safety of operations

- Optimize Airline, ANSP, Airport operations.

- Efficient management during offnominal operations e.g. weather

- Advance Airport Collaborative Decision Making

\section{Benefits}

- ATM optimization based on full view, real time airport information and increased system predictability

- Operational improvements by implementing advances in networking, communications and information management

- Context Awareness - Total view of airport status (facilities/assets, operations, users)

- Improve airport tenant decision making capabilities. 


\section{Project Plan}

\section{Needs Analysis}

- Requirements Development and Analysis

- Concept Architecture Development

\section{Concept Exploration and Definition}

- Communications Link Description/Selection

- Network Architecture

- Architecture Configuration and Test Development

- Architecture Test

\section{Architecture Development and Design}

- Architecture Synthesis

- Architecture Test Development

- Architecture Integration testing

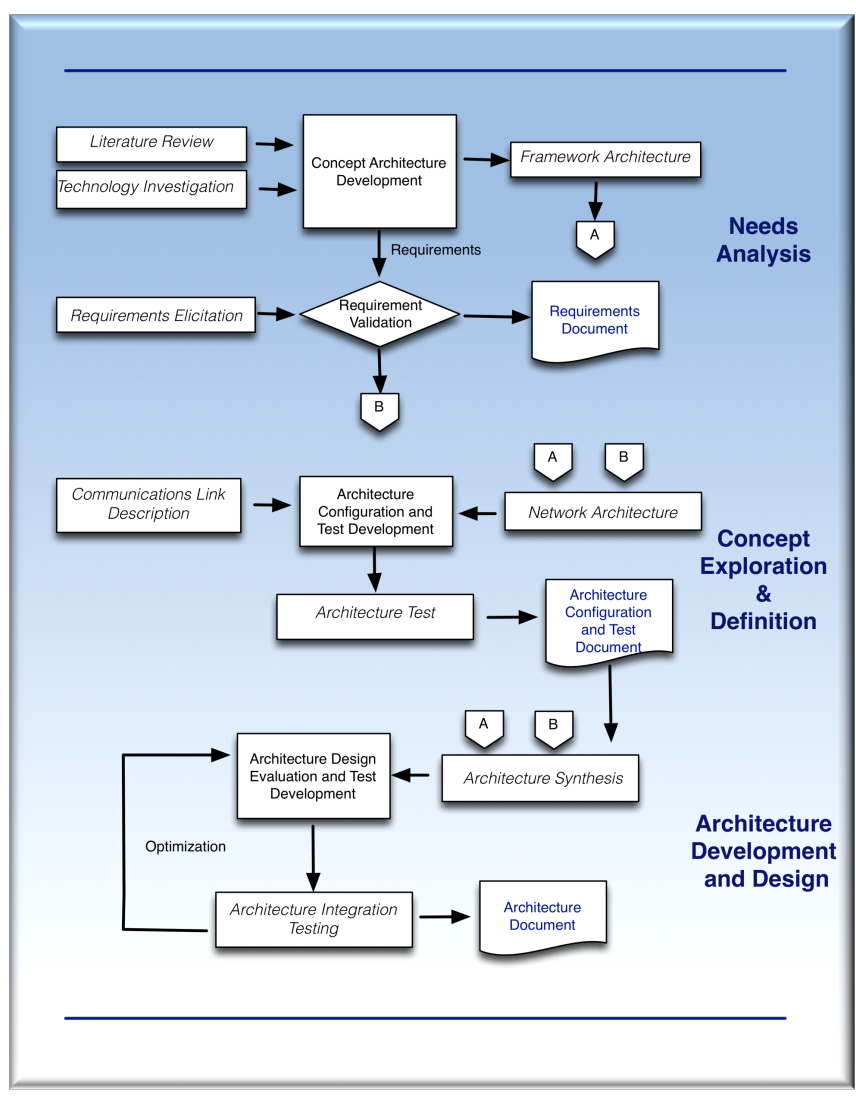




\section{FY-2016 Accomplishments}

\section{Concept Architecture Framework}

- Literature Review

- NextGen Architecture Configuration (Today - 2025)

- Beyond NextGen Architecture Concept

- $\quad$ Airport Authority Operations

- $\quad$ Air Carrier AOC, FOC future operational concepts

- Technology Investigation

- $\quad$ Cloud Computing, Resource Sharing

- Information Centric Networking, Security

- $\quad$ Cognitive Network Ops, Software Defined Network.

- Modern Data Link systems

- Concept Architecture Development

- Model Architecture Definition

Requirements Development and Analysis
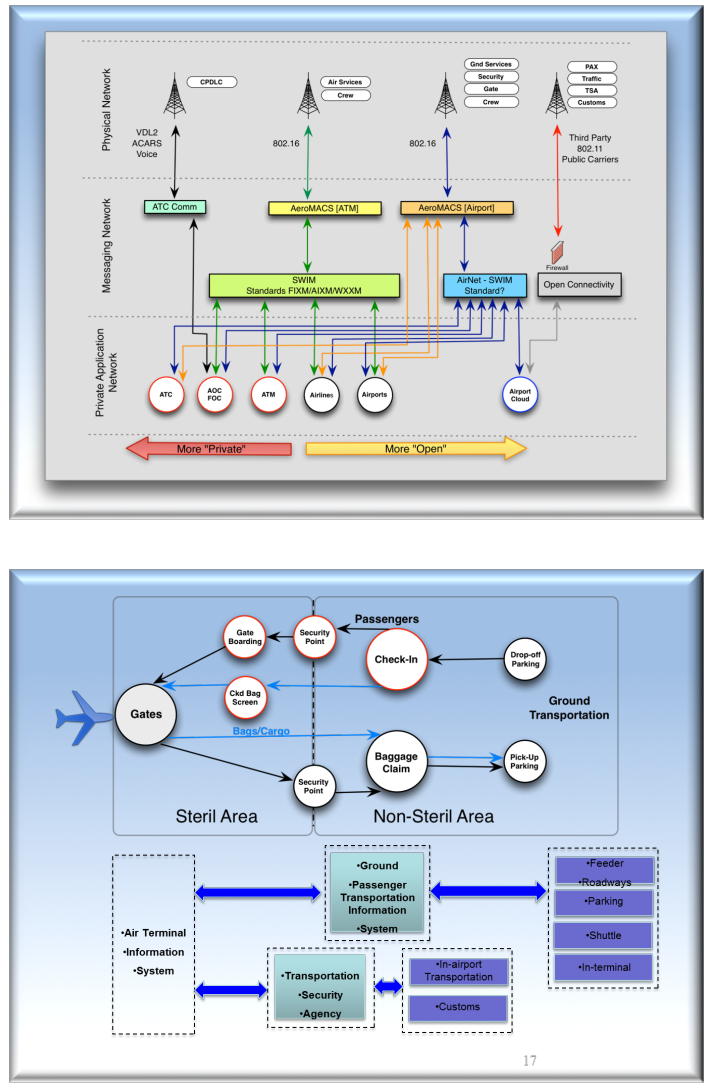


\section{Who are the Stakeholders?}

\section{Airports primarily divided in two areas: Passenger Terminal and Airfield}

- $\quad$ FAA

- Air Traffic

- Technical Operations

- Airlines

- AOC

- Ramp Tower, Baggage, Passenger, Maintenance, etc.

- Aircrew

- Port Authority

- Airfield Ops.

- Terminal Ops.

- Ground handling operations

- Fueling

- Maintenance

- Cabin services

\section{Passenger Terminal}

- TSA

- Customs

- Parking

- Taxi services

- Shuttle Services

- Rail Services

- Customer Services

- People mover Systems

- Local DoT

- Car Rentals

- Gate agent services

- Passengers 


\section{Airport Architecture Concept}




\section{Architecture Approach Options}

- Information exchange through voice communications among principal stakeholders

- New airport network design interconnecting stakeholders

- Interconnect existing stakeholder owned and operated networks

- Develop new Publish/Subscribe Service Oriented Architecture employing cloud services. Stakeholders publish relevant information

- Support of self-healing networks. 


\section{Airport Information Exchange Architecture Framework - Conceptual}

\section{Airport System Information}

- Cloud Implementation

- Publish - Subscribe Service

- Data Center

- Formalized Services

- Web Services

- Airport Exchange Data Model Definition

- Governance Model not in Project

\section{Characteristics}

- High Availability

- Secure

- Scalable

- Resilient

- Real Time Monitoring

- Decouple Publish and Subscribe

- Asynchronous
Publish/Subscribe

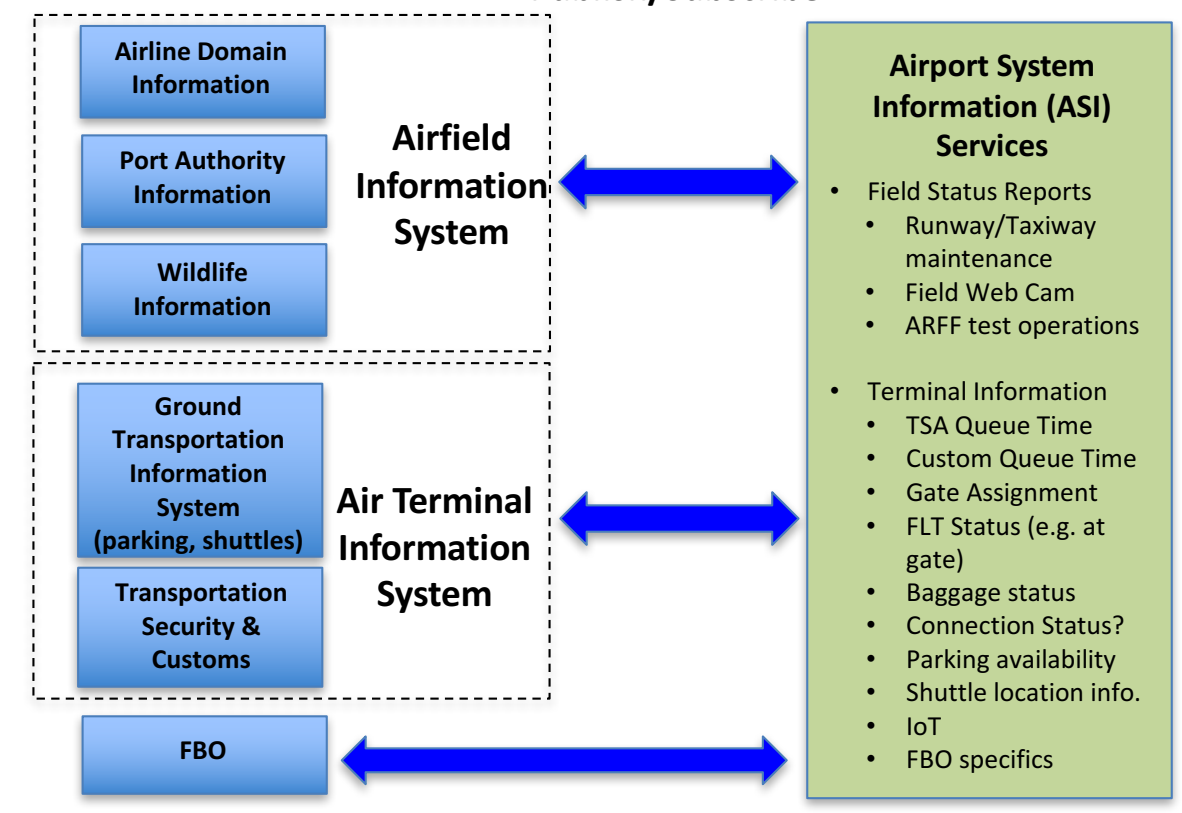

Airfield Information System: Services and information that impact aircraft on time departure/arrival or overall operation of airport surface system

Air Terminal Information System: Services and information impacting passenger's arrival at departure gate. Airport System Information: Repository of relevant airport information supporting ATM/ATC, AOC, FOC, Airports, Passenger 


\section{Benefits and Information Exchange}

\section{Potential Benefits}

Improve on-time Pushback Reduce gate turn around Optimal Departure/Arrival Improve Predictability Increase Airport Capacity Enable Airport CDM Lower Operational Costs Improve Coordination Improve Flow of People through Airport

Improve connection times Gate Assignment Planning

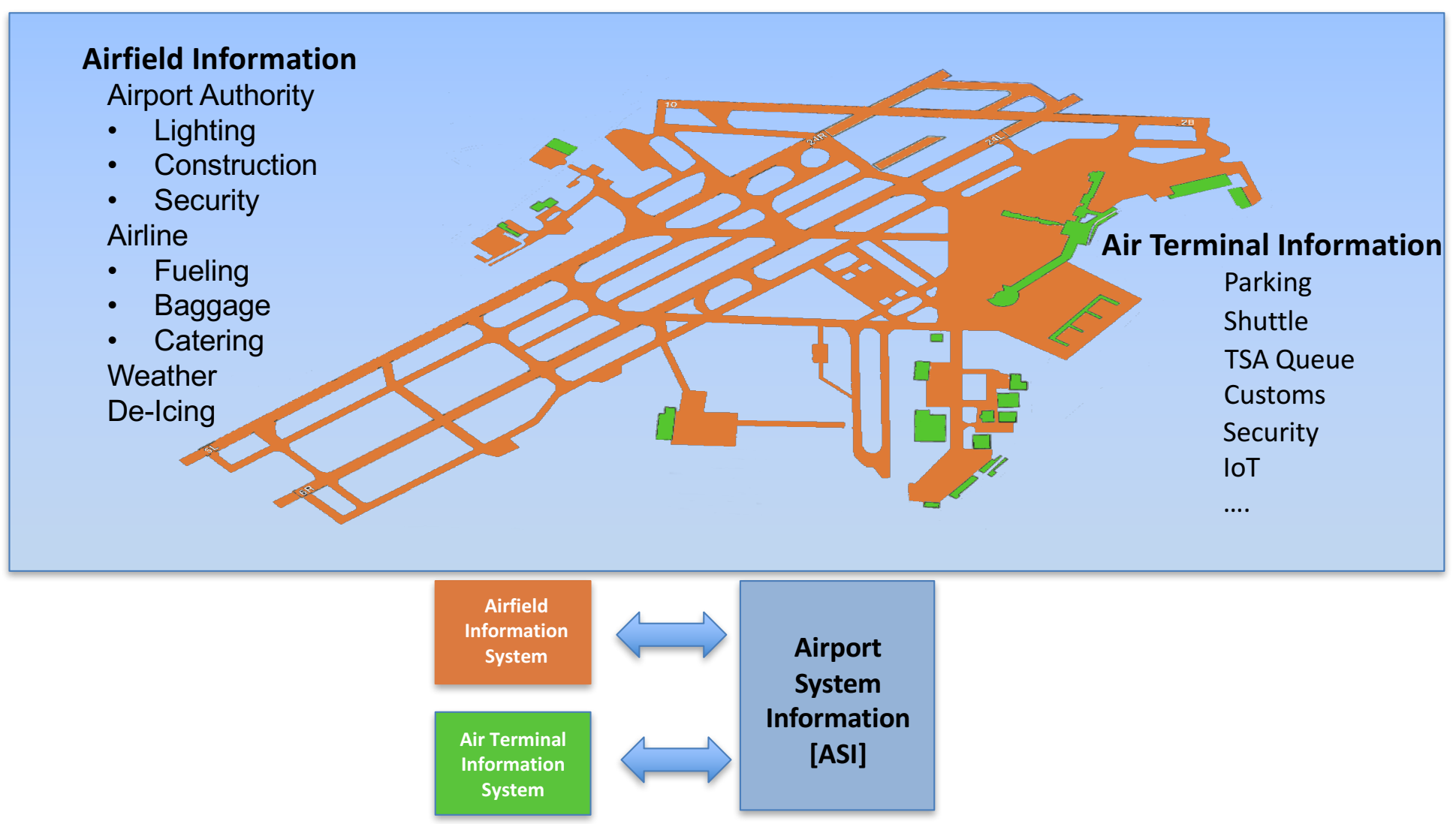

Airport Network Architecture - Facility Information Flow 


\section{Airport Authority Information Exchange - Sample}

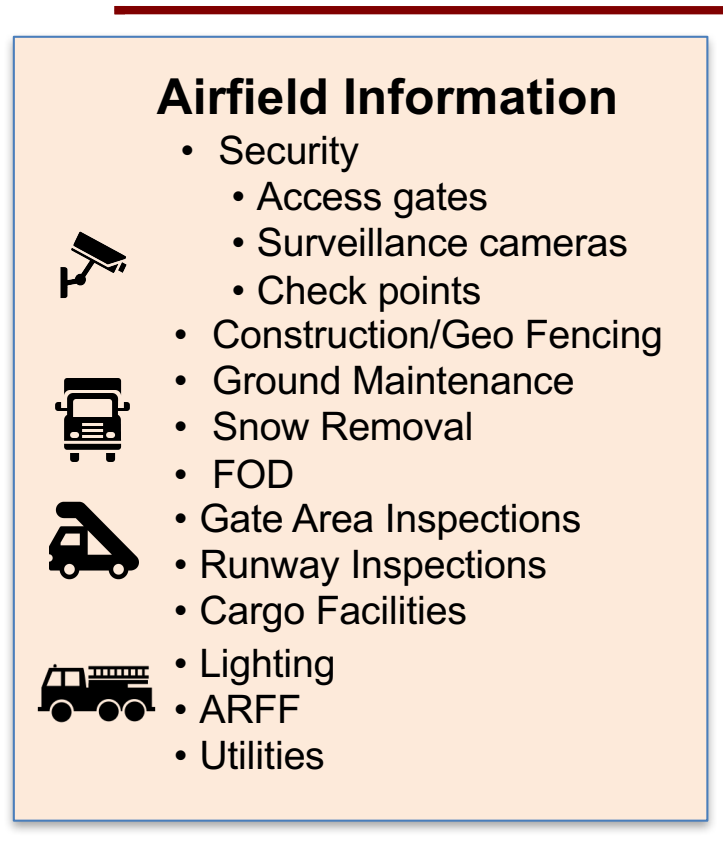

- Wireless Comm.

- Wired Comm.

- IP Connectivity Information Exchange Model \& API

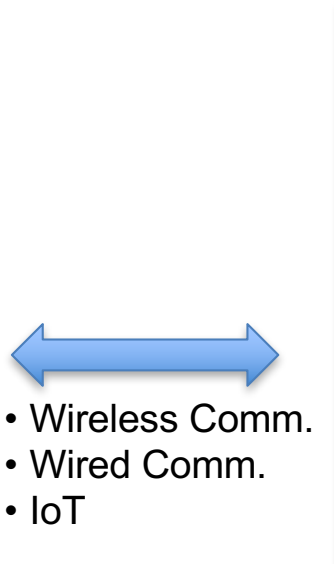

\section{Air Terminal Information}

- Airport Security

- Building Maintenance

- Airport Shuttle

- Airport Parking

- Power Plant

- Heating/Cooling
Information Network

and Processing

(Analytics)

- IoT
Selected Information

Published

\section{Information Consumers}

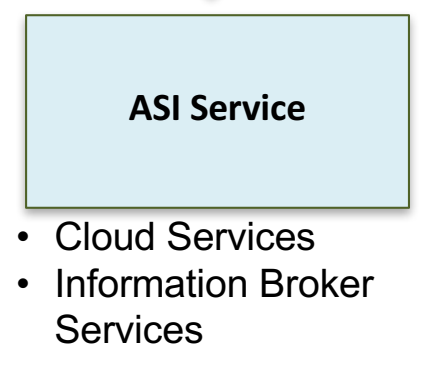

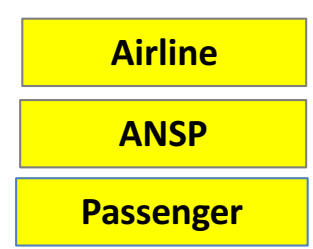

Information Exchange

Model

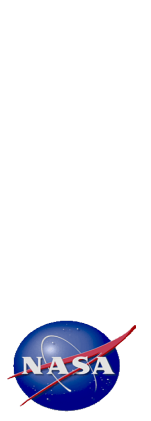




\section{Global Airport Architecture System - Conceptual}

\section{Airport Network System (ANS)} Large scale multi-airport domain network

- Shared brokered network

- Each domain hosts regional airports

- Distributed/federated system

- Data exchange model

- Cloud enabled

- Information offered to:

- ANSP

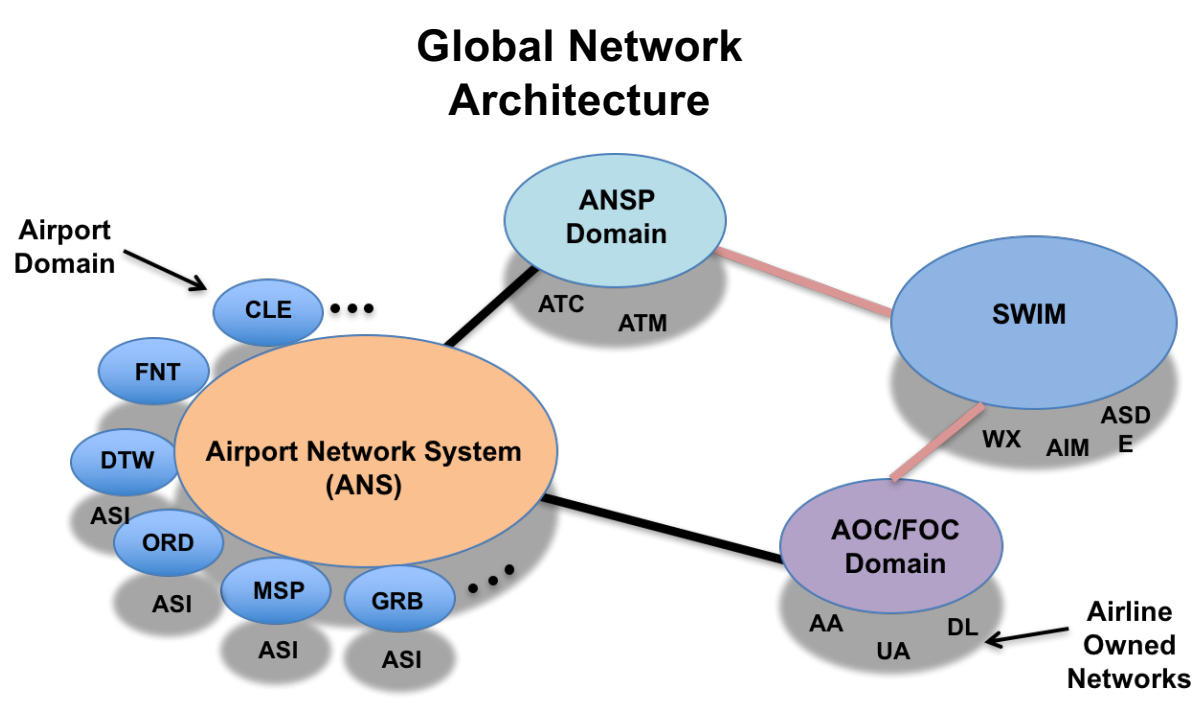

- Airlines

- Cargo

- Airports

- Passengers 


\section{Requirements Development}




\section{Requirement Analysis}

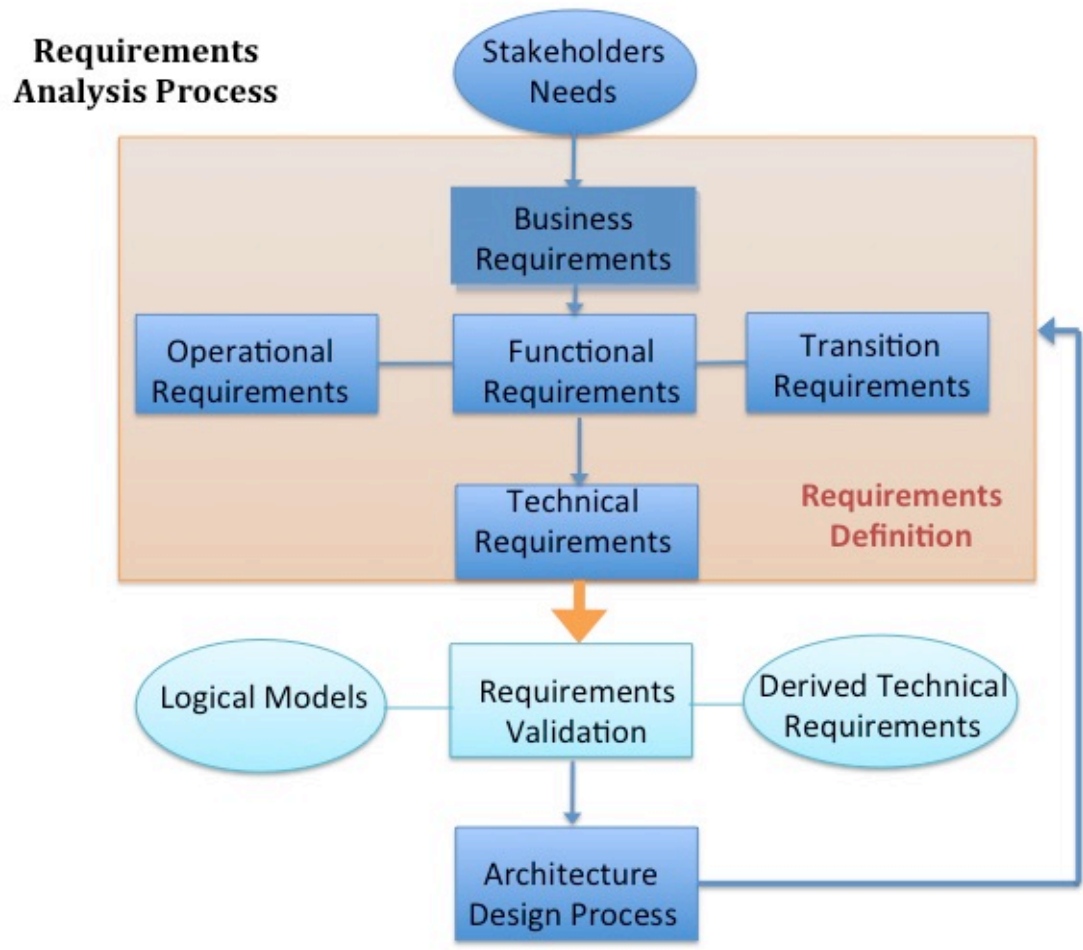




\section{Port Authority Requirements - Sample}

- FAR 139 Safety Inspection

- Navigational Aids System Maintenance

- Signage

- Pavement Management System

- FOD management

- AOA Incident Reporting

- AOA Restricted Area Access Control

- Work Order Generation, Dispatch \& Verification

- Snow Removal Monitoring and Ice Control

- Weather Monitoring

- Wildlife Management System

- Construction Management System

- Vehicle Inspections (outside Air Field)

- Vehicle Inspections (inside Air Field)

- Building room Inspections

- Rules and regulation enforcement

\section{- Requirements}

- Availability: 0.9999

- Duplicity: full duplex

- Latency: 50 msec

- Throughput: 64 kbps

- Symmetry: Full symmetry

- Content: text/light graphics

- Transport Protocol: TCP/IP

- Interface: Ethernet

- Service Recovery: Medium Priority

- Source Equipment: varies

- Sink Equipment: Server 


\section{Use Cases \& Information Flows}




\section{Use case analysis: Use case diagrams}

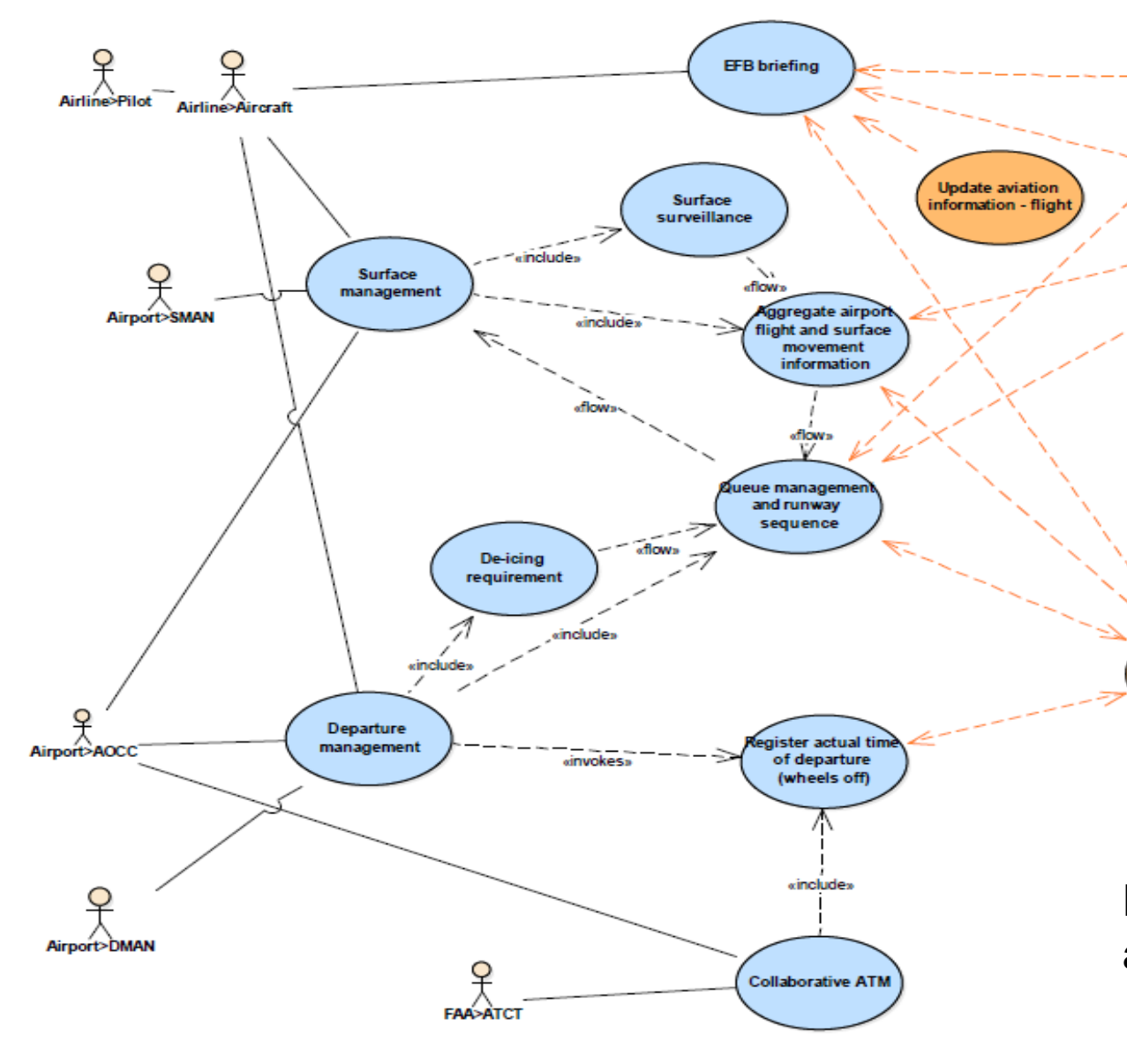

- Identify actors and actions

- Functional relations and dependencies

- No sequential information

- Independent from networking or technology

Example: aircraft departure on airport surface 


\section{Use case analysis: Information flow diagrams}

- Show the sequence of message exchanges required to execute the nominal case of a use case

- Independent from lower level network components
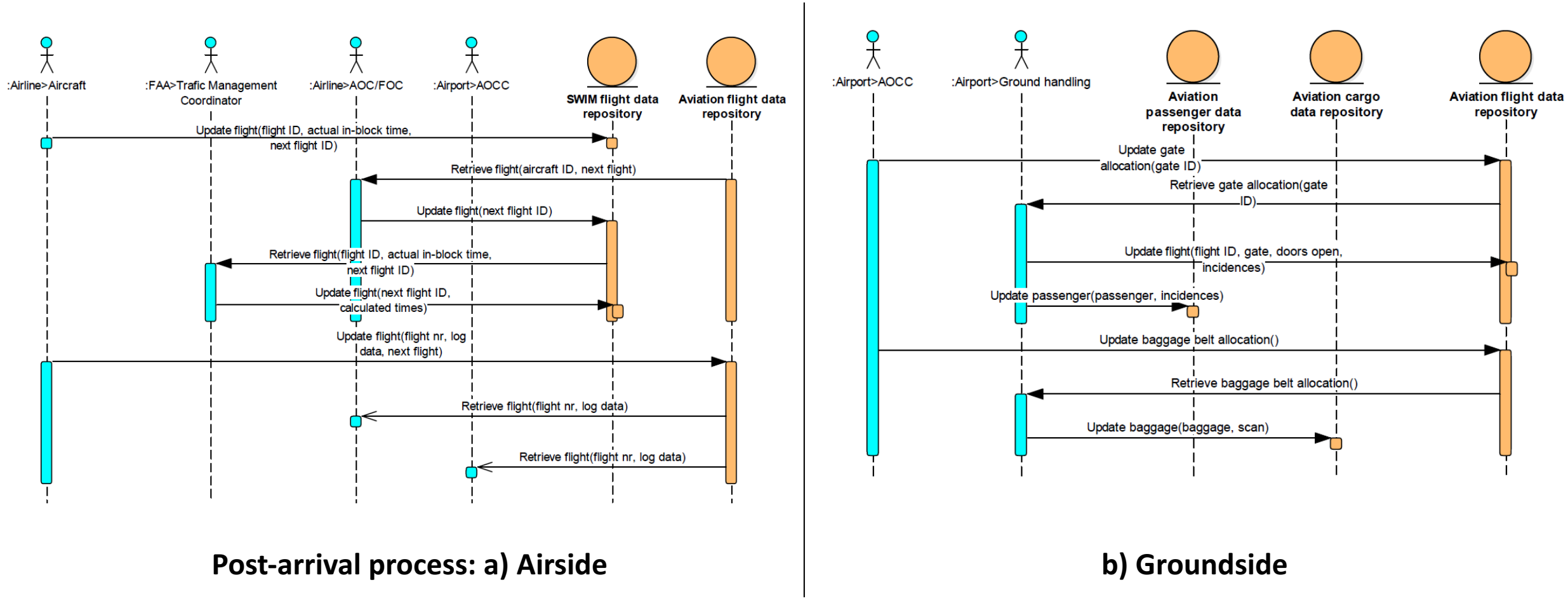

b) Groundside 


\section{Use case analysis: Logical architecture}

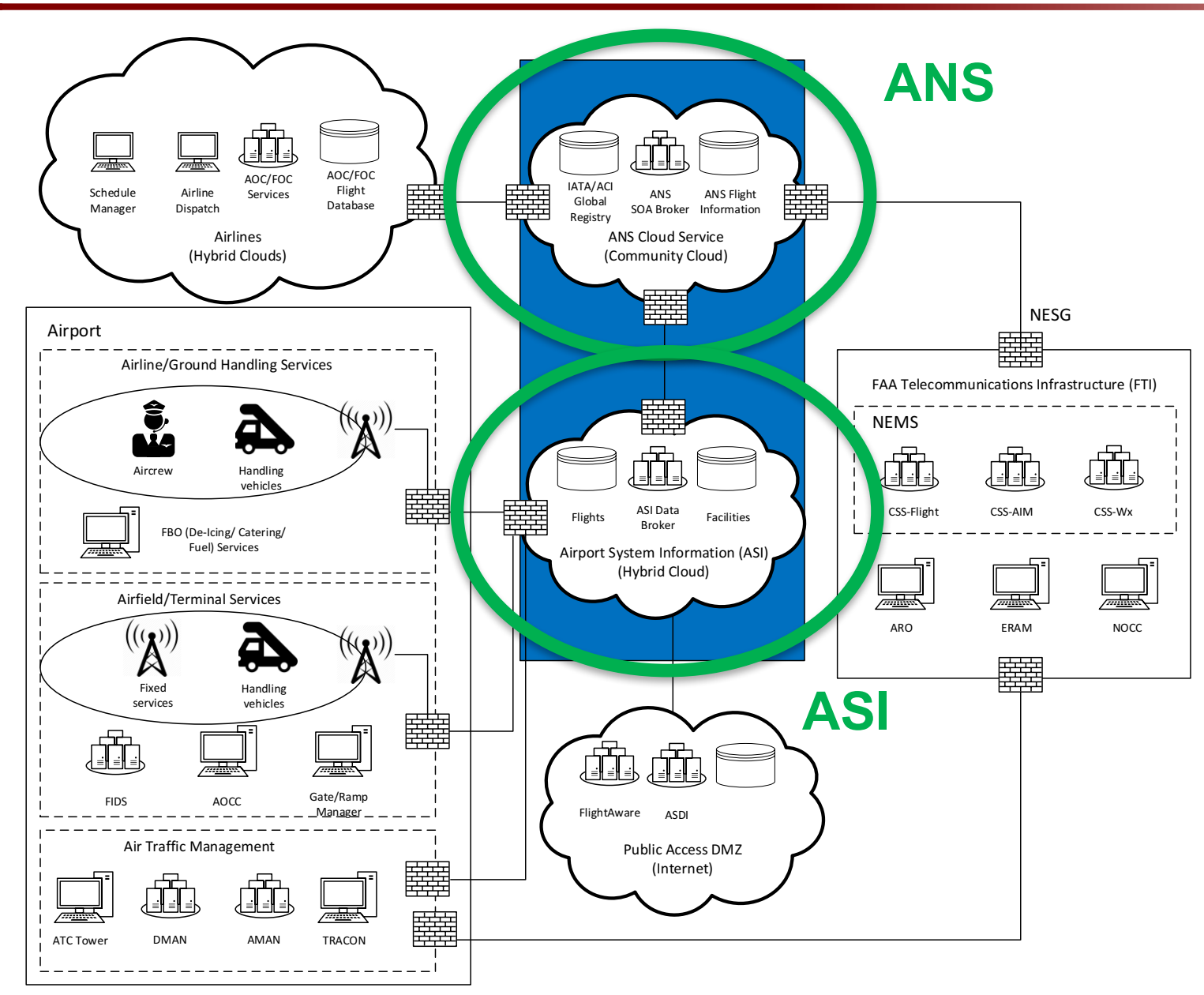

- Defines essential elements and functions to support use cases and information flows

- Data consumers, producers and processors 


\section{Future Work and Conclusion}




\section{Tasks FY-2017}

\section{Concept Exploration Definition}

- Communications Link Evaluation

- Wired and Wireless Communications Technology Identification

- Wired and Wireless Communications Technology Selection

- Wired and Wireless Communications Technical Parameter Definition

- Information Exchange Architecture

- Scenario Development

- Data loading Model Development

- Architecture Development

- Simulation Tool Assessment and Selection 


\section{Conclusion}

- Implementation of information sharing infrastructure and technologies will greatly enhance real time decisionmaking and enable Airport System context awareness.

- Airport Context Awareness will enable airport users to identify who is using services/facilities, what resources are in use, state of resources, operational service limitations, capacity management and others.

- Airport System context awareness will enable Precision Operations 